7. Griffin D, Parsons N, Shaw E, Kulikov Y, Hutchinson C, Thorogood M et al (2014) Operative versus nonoperative treatment for closed, displaced, intraarticular fractures of the calcaneus: Randomised controlled trial. BMJ 349:g4483

8. Pounder NM, Phillips M, Rueger J, Heeckt P (2016) A matter of TRUST: An issue of compliance. BMJ 355(i5351)

\title{
Erratum
}

Unfallchirurg 2017 · 120:272

DOI 10.1007/s00113-017-0320-7

Online publiziert: 24. Februar 2017

(c) Springer Medizin Verlag Berlin 2017

(1.) CrossMark

U. Schweigkofler ${ }^{1} \cdot$ S. Flohé ${ }^{2} \cdot$ R. Hoffmann ${ }^{1} \cdot$ G. Matthes ${ }^{3} \cdot$ T. Paffrath ${ }^{4} \cdot$ C. Wölfl . $^{5}$

M. Fischer ${ }^{6,7} \cdot$ E. Kehrberger ${ }^{6,8} \cdot$ H. Marung ${ }^{9,10} \cdot$ H. Moecke ${ }^{10} \cdot$ S. Prückner ${ }^{11}$.

B. Urban ${ }^{11} \cdot \mathrm{H}$. Trentzsch ${ }^{11} \cdot$ Fachexperten der Eckpunktepapier-Konsensusgruppe

'Unfallchirurgie und Orthopädische Chirurgie, BG Unfallklinik Frankfurt am Main gGmbH, Frankfurt am Main, Deutschland

${ }^{2}$ Klinik für Unfallchirurgie, Orthopädie und Handchirurgie, Städtisches Klinikum Solingen gemeinnützige $\mathrm{GmbH}$, Solingen, Deutschland

${ }^{3}$ Klinik für Unfallchirurgie und Orthopädie, Unfallkrankenhaus Berlin, Berlin, Deutschland

${ }^{4}$ Klinik für Unfallchirurgie, Orthopädie \& Sporttraumatologie, Lehrstuhl für Unfallchirurgie \& Orthopädie, Klinikum der Privaten UniversitätWitten/Herdecke, Köln, Deutschland

${ }^{5}$ Klinik für Orthopädie, Unfallchirurgie und Sporttraumatologie, Krankenhaus Hetzelstift, Neustadt/Weinstraße, Deutschland

${ }^{6}$ Arbeitsgemeinschaft Südwestdeutscher Notärzte e. V. (AGSWN), Filderstadt, Deutschland

${ }^{7}$ Klinik für Anästhesiologie, Operative Intensivmedizin, Notfallmedizin und Schmerztherapie, Klinik am Eichert, Göppingen, Deutschland

${ }^{8}$ Klinik für Anästhesiologie und operative Intensivmedizin, Kreiskliniken Esslingen, ParacelsusKrankenhaus Ruit, Ostfildern, Deutschland

${ }^{9}$ Institut für Rettungs- und Notfallmedizin, Universitätsklinikum Schleswig-Holstein, Kiel, Deutschland

${ }^{10}$ Institut für Notfallmedizin (IfN), Asklepios Klinikum Harburg, Hamburg, Deutschland

${ }^{11}$ Institut für Notfallmedizin und Medizinmanagement (INM), Klinikum der Universität München, LudwigMaximilians-Universität München, München, Deutschland

\section{Erratum zu: Tracerdiagnose Schwerverletzte/Polytrauma im Eckpunktepapier 2016}

\section{Erratum zu: \\ Unfallchirurg 2016 \\ DOI $10.1007 / \mathrm{s} 00113-016-0256-3$}

Bei der Korrespondenzadresse von Dr. H. Trentzsch ist uns bei diesem Beitrag leider ein Fehler unterlaufen. Wir bitten die korrekte Adresse zu beachten und den Fehler zu entschuldigen.

Die Redaktion

\section{Korrespondenzadresse}

\section{Dr. H. Trentzsch}

Institut für Notfallmedizin und Medizinmanagement (INM), Klinikum der Universität München, Ludwig-Maximilians-Universität München

Schillerstraße 53, 80336 München, Deutschland heiko.trentzsch@med.uni-muenchen.de 